\title{
A Boundary Perturbation Method for Recovering Interface Shapes in Layered Media
}

\author{
Alison Malcolm \\ Department of Earth, Atmospheric, and Planetary Sciences \\ Massachusetts Institute of Technology \\ Cambridge, MA 02139 \\ David P. Nicholls \\ Department of Mathematics, Statistics, and Computer Science \\ University of Illinois at Chicago \\ Chicago, IL 60607
}

July 12,2011

\begin{abstract}
The scattering of linear acoustic radiation by a periodic layered structure is a fundamental model in the geosciences as it closely approximates the propagation of pressure waves in the earth's crust. In this contribution the authors describe new algorithms for (1.) the forward problem of prescribing incident radiation and, given known structure, determining the scattered field, and (2.) the inverse problem of approximating the form of the structure given prescribed incident radiation and measured scattered data. Each of these algorithms is based upon a novel statement of the problem in terms of boundary integral operators (Dirichlet-Neumann operators), and a Boundary Perturbation algorithm (the Method of Operator Expansions) for their evaluation. Detailed formulas and numerical simulations are presented to demonstrate the utility of these new approaches.
\end{abstract}

\section{Introduction}

The interior of the earth's crust can effectively be modeled as a layered media: Largely homogeneous blocks of material separated by sharp interfaces across which material properties change discontinuously. With such a model in mind one can pose two important and related questions: (1.) Given knowledge of the material properties of the layers and the shapes of the interfaces, can one compute scattering returns from such a structure given incident radiation? (2.) Specifying incident radiation and measuring scattered waves, can one deduce information about material properties and interface shapes within the layered media? In this paper we take up both questions (the "forward" problem, (1.), and the "inverse" problem (2.)) and propose novel algorithms for each. These algorithms are based upon a new formulation of the problem in terms of Dirichlet-Neumann operators (DNOs), and convenient Boundary Perturbation (BP) formulas for their simulation.

Unsurprisingly, the full complement of classical numerical methods have been brought to bear upon both the forward and inverse problems we mention above. The Finite Difference (FDM) [MRE07, Pra90], Finite Element (FEM) [Zie77, KFI04], and Spectral Element (SEM) [KT02a, KT02b] methods have been implemented but suffer from the fact that they 
discretize the full volume of the model incurring significant cost, and the difficulty of faithful enforcement of far-field boundary conditions. A compelling alternative are surface methods [SSPRCP89, Bou03] (e.g. Boundary Integral Methods or Boundary Element Methods) which only require a discretization of the layer interfaces (rather than the whole structure) and which, due to the choice of the Green's function, enforce the far-field boundary condition exactly. However, these methods, while capable of delivering high-accuracy solutions, must not only utilize specially designed quadrature rules which respect the singularities in the Green's function, but also generate a dense system of linear equations to be solved which require carefully designed preconditioned iterative methods (with accelerated matrix-vector products, e.g., by the Fast-Multipole Method [GR87]).

The literature on methods for the inverse problem is as vast as that for the forward problem, occupying hundreds of books and thousands of papers (the text of Colton \& Kress [CK98] is an excellent starting point). Interestingly, most of the work has concerned the bounded-obstacle problem, but for the recovery of interface shapes in layered media we point out some recent work based upon classical integral formulations and solution of the resulting (nonlinear and ill-conditioned) equations [KT00, AKY06, LG11]. For a more extensive review, we refer the interested reader to the bibliographies of these.

Here we propose a Boundary Perturbation method for both the forward and inverse problem for irregularly shaped periodic layered media. Like Boundary Integral/Equation Methods, our approach requires only the discretization of the layer interfaces while it avoids not only the need for specialized quadrature rules but also the solution of dense linear systems. Our approach is a generalization of the "Method of Operator Expansions" (OE) of Milder [Mil91a, Mil91b, MS91, MS92, Mil96b, Mil96a] which we use precisely because the interface shapes appear so explicitly in these formulations making them particularly appealing for the development of an inversion algorithm. For a generalization of the closely related "Method of Field Expansions" (FE) described by Bruno \& Reitich [BR92, BR93a, BR93b, BR93c] for dielectric structures with multiple layers (denoted there the "Method of Variation of Boundaries"), we refer the interested reader to the authors' recent publication [MN10].

As with the OE method as it was originally designed by Milder, our new approach is spectrally accurate (i.e., has convergence rates faster than any polynomial order) due to both the analyticity of the scattered fields with respect to boundary perturbation, and the optimal choice of spatial basis functions which arise naturally in the methodology. Our inversion strategy is inspired by the work of Nicholls \& Taber [NT08, NT09] on the recovery of topography shape under a layer of an ideal fluid (e.g. the ocean) which also uses the explicit nature of the OE formulas to great effect.

The organization of the paper is as follows: In $\S 2$ we recall the governing equations. In $\S 3$ we discuss considerations of the forward problem, including a new algorithm for the forward problem ( $\$ 3.1)$ and formulas for Taylor series coefficients of the relevant boundary operators $(\S \S 3.2,3.3,3.4)$. We also present the exact formula in the flat interface case $(\S 3.5)$ and a representative numerical result for a non-trivial interface ( $\S 3.6)$. In $\S 4$ we outline our new methods for solving the inverse problem, including both an iteration-free (linear) algorithm ( $\S 4.1$ ) and an iterative (nonlinear) method (§ 4.2); numerical results are presented in $\S 4.3$. 


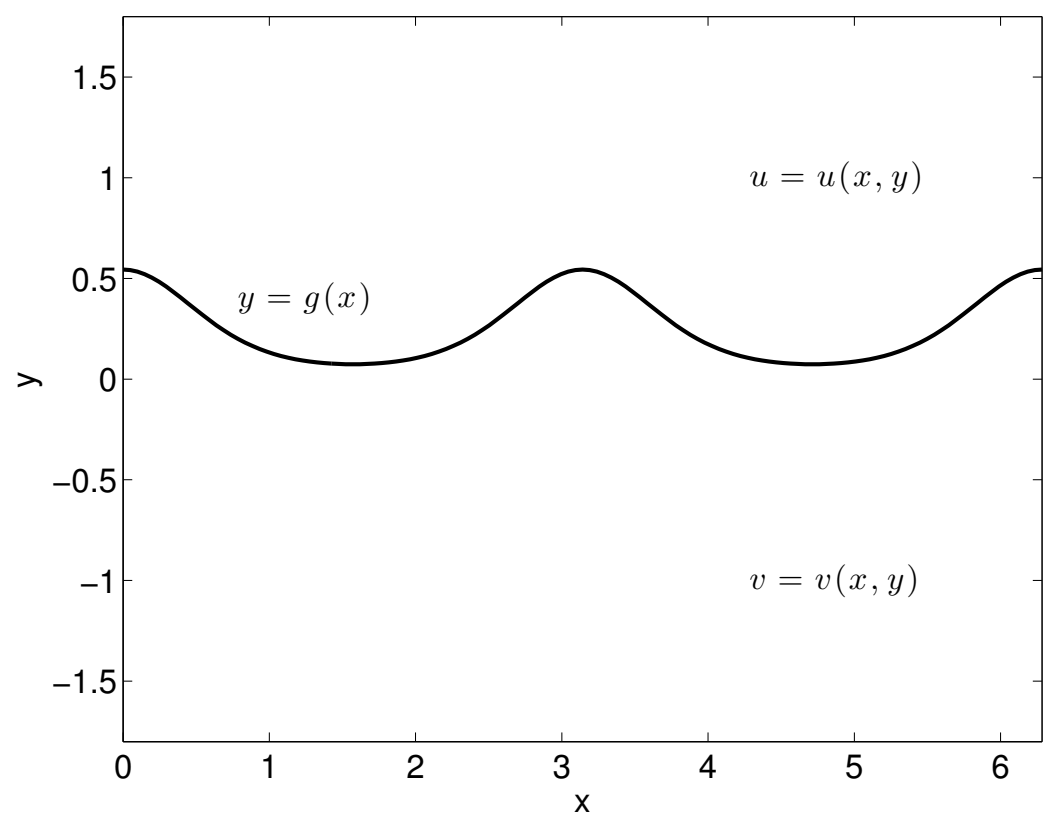

Figure 1: Problem configuration with layer boundary (solid line); here $g(x)=$ $0.2 \exp (\cos (2 x))$.

\section{Governing Equations}

It is well-known that the (reduced) scattered pressure inside a $d$-periodic structure satisfies the Helmholtz equation with illumination conditions at the interface, and outgoing wave conditions at positive and negative infinity. More precisely, we define the domains

$$
S_{u}=\{(x, y) \mid y>g(x)\}, \quad S_{v}=\{(x, y) \mid y<g(x)\}, \quad g(x+d)=g(x),
$$

with (upward pointing) normal

$$
N=\left(-\partial_{x} g, 1\right)^{T}
$$

see Figure 1. Both domains are constant-density acoustic media with velocities $c_{j}(j=u, v)$; we assume that plane-wave radiation of wavenumber $(\alpha,-\beta)=\left(\alpha,-\beta_{u}\right)$ is incident upon the structure from above:

$$
\mathbf{u}(x, y, t)=e^{-i \omega t} e^{i\left(\alpha x-\beta_{u} y\right)}=e^{-i \omega t} u_{i}(x, y) .
$$

With these specifications we can define in each layer the parameter $k_{j}=\omega / c_{j}$ which characterizes both the properties of the material and the frequency of radiation in the structure. If the reduced scattered fields (i.e., the full scattered fields with the periodic time dependence factored out) in $S_{u}$ and $S_{v}$ are respectively denoted $\{u, v\}=\{u(x, y), v(x, y)\}$ then these functions will be quasiperiodic [Pet80]

$$
u(x+d, y)=e^{i \alpha d} u(x, y), \quad v(x+d, y)=e^{i \alpha d} v(x, y),
$$


and the system of partial differential equations to be solved are:

$$
\begin{array}{ll}
\Delta u+k_{u}^{2} u=0 & y>g(x) \\
\mathcal{B}\{u\}=0 & y \rightarrow \infty \\
\Delta v+k_{v}^{2} v=0 & y<g(x) \\
\mathcal{B}\{v\}=0 & y \rightarrow-\infty \\
u-v=\zeta, \quad \partial_{N}(u-v)=\psi & y=g(x),
\end{array}
$$

where

$$
\begin{aligned}
\zeta(x) & :=-u_{i}(x, g(x))=-e^{i\left(\alpha x-\beta_{u} g(x)\right)} \\
\psi(x) & :=-\left[\partial_{N} u_{i}(x, y)\right]_{y=g(x)}=\left(i \beta_{u}+i \alpha\left(\partial_{x} g\right)\right) e^{i\left(\alpha x-\beta_{u} g(x)\right)} .
\end{aligned}
$$

In these equations the operator $\mathcal{B}$ enforces the condition that scattered solutions must either be "outgoing" (upward in $S_{u}$ and downward in $S_{v}$ ) if they are propagating, or "decaying" if they are evanescent. We make this "Outgoing Wave Condition" (OWC) [Pet80] more precise in the Fourier series expression for the exact solution, see (2.3) below.

The quasiperiodic solutions of the Helmholtz equations- (2.2a) \& (2.2c)-and the OWCs- $(2.2 \mathrm{~b}) \&(2.2 \mathrm{~d})$ - are given by [Pet80]

$$
\begin{aligned}
& u(x, y)=\sum_{p=-\infty}^{\infty} a_{p} \exp \left(i\left(\alpha_{p} x+\beta_{u, p} y\right)\right) \\
& v(x, y)=\sum_{p=-\infty}^{\infty} b_{p} \exp \left(i\left(\alpha_{p} x-\beta_{v, p} y\right)\right)
\end{aligned}
$$

where the OWC mandates that we choose the positive sign in front of $\beta_{u, p}$ in (2.3a) and the negative sign in front of $\beta_{v, p}$ in $(2.3 \mathrm{~b})$. These formulas are valid provided that $(x, y)$ are outside the grooves, i.e.

$$
(x, y) \in\left\{y>|g|_{L^{\infty}}\right\} \cup\left\{y<-|g|_{L^{\infty}}\right\} .
$$

In these equations

$$
\alpha_{p}=\alpha+(2 \pi / d) p, \quad \beta_{j, p}= \begin{cases}\sqrt{k_{j}^{2}-\alpha_{p}^{2}} & \alpha_{p}^{2}<k_{j}^{2} \\ i \sqrt{\alpha_{p}^{2}-k_{j}^{2}} & \alpha_{p}^{2}>k_{j}^{2}\end{cases}
$$

$j=u, v$ and $d$ is the period of the structure. Again, the OWC determines the choice of sign for $\beta_{j, p}$ in the evanescent case $\alpha_{p}^{2}>k_{j}^{2}$.

\section{Forward Problem}

For the forward problem we specify the grating $g(x)$ and the Dirichlet and Neumann data from the incident radiation: $\zeta(x)$ and $\psi(x)$. From this we should produce the scattered fields $u(x, y)$ and $v(x, y)$. However, it is not difficult to deduce that if we recover the Dirichlet and Neumann traces of $u$ and $v$

$$
\begin{aligned}
U(x):=u(x, g(x)), & V(x):=v(x, g(x)), \\
U^{\prime}(x):=\left(\partial_{N} u\right)(x, g(x)), & V^{\prime}(x):=\left(\partial_{N} v\right)(x, g(x)),
\end{aligned}
$$


then integral formulas will tell us $u$ and $v$ everywhere.

Furthermore, if we define the Dirichlet-Neumann Operators (DNOs)

$$
G(g)[U(x)]:=U^{\prime}(x), \quad H(g)[V(x)]:=V^{\prime}(x),
$$

then it suffices to find simply the Dirichlet traces $U$ and $V$. As the DNOs encapsulate the solution of the Helmholtz equations and the OWCs, it is not difficult to see that (2.2) are equivalent to the surface equations

$$
\begin{aligned}
& U-V=\zeta \\
& G[U]-H[V]=\psi .
\end{aligned}
$$

This can be simplified in a number of ways, but one which is convenient for our current purposes uses the first equation to solve for $V, V=U-\zeta$, which is then inserted into the second equation yielding

$$
(G-H)[U]=\psi-H[\zeta] .
$$

As the boundary quantity $U$ will be inconvenient or impossible to recover, we note that an alternative quantity to recover is the "far field" data

$$
\tilde{u}(x):=u(x, a),
$$

for some $a>|g|_{L^{\infty}}$. We point out that there is some ambiguity in the term "far field" as some authors use this to characterize the propagating modes solely, whereas we use it to mean "away" from the grating (where the evanescent modes will have exponentially small, but nonzero, effect). As we comment later (§ 3.3), the location of the far-field hyperplane $y=a$ has a strong influence on the behavior of our inversion algorithm. This value encodes the inherent ill-posedness of our recovery scheme and as a increases, the accuracy of our method deteriorates rather rapidly.

If we define the "backward propagator" $L$ by

$$
L(g)[\tilde{u}(x)]:=U(x),
$$

then we can replace (3.2) with

$$
(G-H)[L[\tilde{u}]]=\psi-H[\zeta],
$$

or, for use with our inversion algorithms,

$$
0=Q(g)[\tilde{u}]:=(G-H)[L[\tilde{u}]]-\psi+H[\zeta] .
$$

\subsection{A New Algorithm for the Forward Problem}

We propose a perturbative approach to the solution of (3.3) based upon the assumption $g(x)=\varepsilon f(x)$ where, a priori, $\varepsilon$ is assumed small. If this is the case then it can be shown that the data $\{\zeta, \psi\}$ and operators $\{G, H, L\}$ depend analytically upon $\varepsilon$ so that

$$
\begin{gathered}
\zeta=\zeta(x ; \varepsilon)=\sum_{n=0}^{\infty} \zeta_{n}(x) \varepsilon^{n}, \quad \psi=\psi(x ; \varepsilon)=\sum_{n=0}^{\infty} \psi_{n}(x) \varepsilon^{n} \\
G=G(\varepsilon f)=\sum_{n=0}^{\infty} G_{n}(f) \varepsilon^{n}, \quad H=H(\varepsilon f)=\sum_{n=0}^{\infty} H_{n}(f) \varepsilon^{n} \\
L=L(\varepsilon f)=\sum_{n=0}^{\infty} L_{n}(f) \varepsilon^{n}
\end{gathered}
$$


and we assume

$$
\tilde{u}=\tilde{u}(x ; \varepsilon)=\sum_{n=0}^{\infty} \tilde{u}_{n} \varepsilon^{n} .
$$

A rigorous justification for these expansions can be found in the work of Coifman \& Meyer [CM85], Craig, Schanz, \& Sulem [CSS97], and the authors (in collaboration with Reitich and $\mathrm{Hu}$ ) [NR01, NR03, NR04b, HN05, HN10]. Inserting this into (3.3) we see that

$$
\left(\sum_{n=0}^{\infty} \varepsilon^{n}\left(G_{n}-H_{n}\right)\right)\left[\left(\sum_{s=0}^{\infty} \varepsilon^{s} L_{s}\right)\left[\sum_{m=0}^{\infty} \tilde{u}_{m} \varepsilon^{m}\right]\right]=\sum_{n=0}^{\infty} \psi_{n} \varepsilon^{n}-\left(\sum_{n=0}^{\infty} \varepsilon^{n} H_{n}\right)\left[\sum_{m=0}^{\infty} \zeta_{m} \varepsilon^{m}\right] .
$$

At order $\mathcal{O}\left(\varepsilon^{0}\right)$

$$
\tilde{u}_{0}=L_{0}^{-1}\left[\left(G_{0}-H_{0}\right)^{-1}\left[\psi_{0}-H_{0}\left[\zeta_{0}\right]\right]\right]
$$

while at order $\mathcal{O}\left(\varepsilon^{n}\right)$

$$
\sum_{s=0}^{n} \sum_{m=0}^{s}\left(G_{n-s}-H_{n-s}\right)\left[L_{s-m}\left[\tilde{u}_{m}\right]\right]=\psi_{n}-\sum_{m=0}^{n} H_{n-m}\left[\zeta_{m}\right] .
$$

Solving for $\tilde{u}_{n}$,

$$
\begin{aligned}
\tilde{u}_{n}=L_{0}^{-1}\left(G_{0}-H_{0}\right)^{-1}\left\{\psi_{n}-\sum_{m=0}^{n} H_{n-m}\left[\zeta_{m}\right]-\right. & \sum_{s=0}^{n-1} \sum_{m=0}^{s}\left(G_{n-s}-H_{n-s}\right)\left[L_{s-m}\left[\tilde{u}_{m}\right]\right] \\
& \left.-\sum_{m=0}^{n-1}\left(G_{0}-H_{0}\right)\left[L_{n-m}\left[\tilde{u}_{m}\right]\right]\right\} .
\end{aligned}
$$

Note that at every perturbation order in this approach we repeatedly invert the common operator $\left(G_{0}-H_{0}\right) L_{0}$ which is, in Fourier space, diagonal and can, therefore, be accomplished very rapidly.

\subsection{Expansions: Surface Data}

The key to both our forward and inverse algorithms are convenient, high order formulas for the functions $\zeta_{n}$ and $\psi_{n}$, and the operators $G_{n}, H_{n}$, and $L_{n}$. We begin with $\zeta$ :

$$
\zeta(x ; \varepsilon)=-e^{i\left(\alpha x-\beta_{u} \varepsilon f(x)\right)}=-e^{i \alpha x} \sum_{n=0}^{\infty} F_{n}(x)\left(-i \beta_{u}\right)^{n} \varepsilon^{n},
$$

where $F_{n}(x):=f(x)^{n} / n$ !. Thus

$$
\zeta_{n}=-e^{i \alpha x} F_{n}(x)\left(-i \beta_{u}\right)^{n} .
$$

Similarly, for $\psi$ we have

$$
\begin{aligned}
\psi(x) & =\left(i \beta_{u}+i \alpha \varepsilon\left(\partial_{x} f\right)\right) e^{i\left(\alpha x-\beta_{u} \varepsilon f(x)\right)} \\
& =e^{i \alpha x}\left(i \beta_{u} \sum_{n=0}^{\infty} F_{n}(x)\left(-i \beta_{u}\right)^{n} \varepsilon^{n}+i \alpha \varepsilon\left(\partial_{x} f\right) \sum_{n=0}^{\infty} F_{n}(x)\left(-i \beta_{u}\right)^{n} \varepsilon^{n}\right) .
\end{aligned}
$$

So

$$
\psi_{n}=e^{i \alpha x}\left(-F_{n}(x)\left(-i \beta_{u}\right)^{n+1}+\left(\partial_{x} f\right) F_{n-1}(x)(i \alpha)\left(-i \beta_{u}\right)^{n-1}\right) .
$$




\subsection{Expansions: Backward Propagator Operator}

The operators $\{L, G, H\}$ are a bit more involved and we will use the method of "Operator Expansions" (OE) [Mil91a, CS93, NR04a] to find the action of $\left\{L_{n}, G_{n}, H_{n}\right\}$ on a Fourier basis function which, of course, leads to its action on any $L^{2}$ function. To begin we consider the operator $L$ which maps the far-field data $\tilde{u}$ to the surface data $U$. The function

$$
u_{p}(x, y)=e^{i\left(\alpha_{p} x+\beta_{u, p}(y-a)\right)}
$$

satisfies Helmholtz's equation and the outgoing wave condition in the upper material. We can insert this into the definition of the operator $L$ giving

$$
L(g)\left[u_{p}(x, a)\right]=u_{p}(x, g(x))
$$

or

$$
L(g)\left[e^{i \alpha_{p} x}\right]=e^{i\left(\alpha_{p} x+\beta_{u, p}(g(x)-a)\right)} .
$$

Setting $g(x)=\varepsilon f(x)$, and expanding $L$ and the exponential in Taylor series reveals

$$
\left(\sum_{n=0}^{\infty} \varepsilon^{n} L_{n}(f)\right)\left[e^{i \alpha_{p} x}\right]=e^{i \alpha_{p} x} e^{-i \beta_{u, p} a} \sum_{n=0}^{\infty} F_{n}(x)\left(i \beta_{u, p}\right)^{n} \varepsilon^{n} .
$$

At order $\mathcal{O}\left(\varepsilon^{0}\right)$ we discover

$$
L_{0}\left[e^{i \alpha_{p} x}\right]=e^{-i \beta_{u, p} a} e^{i \alpha_{p} x}=e^{-i \beta_{u, D} a} e^{i \alpha_{p} x}
$$

where we have introduced a Fourier multiplier

$$
m(D)[\xi(x)]:=\sum_{p=-\infty}^{\infty} m(p) \hat{\xi}_{p} e^{i \alpha_{p}} .
$$

Using the fact that any $\alpha$-quasiperiodic $L^{2}$ function can be expressed via its Fourier series we deduce that

$$
L_{0}[\xi]=e^{-i \beta_{u, D} a} \xi=\sum_{p=-\infty}^{\infty} e^{-i \beta_{u, p} a} \hat{\xi}_{p} e^{i \alpha_{p}} .
$$

At order $\mathcal{O}\left(\varepsilon^{n}\right)$ we find

$$
L_{n}(f)\left[e^{i \alpha_{p} x}\right]=e^{i \alpha_{p} x} e^{-i \beta_{u, p} a} F_{n}(x)\left(i \beta_{u, p}\right)^{n}=F_{n}(x) e^{-i \beta_{u, D} a}\left(i \beta_{u, D}\right)^{n} e^{i \alpha_{p} x},
$$

so that

$$
L_{n}(f)[\xi]=F_{n}(x) e^{-i \beta_{u, D} a}\left(i \beta_{u, D}\right)^{n} \xi=F_{n}(x) L_{0}\left(i \beta_{u, D}\right)^{n} \xi=F_{n}(x)\left(i \beta_{u, D}\right)^{n} L_{0} \xi .
$$

Remark. We will soon introduce an inversion algorithm for the interface shape $g$ based upon the formulae presented in these sections. A fundamental feature of such problems is severe ill-posedness and we point out that this is reflected in the operator $L_{0}$. For $p$ corresponding to propagating waves ( $p$ sufficiently small) we have chosen $\beta_{u, p}$ real so that the Fourier multiplier $\exp \left(-i \beta_{u, p} a\right)$ is of modulus one. However, for $p$ corresponding to evanescent modes ( $p$ large) $\beta_{u, p}$ is purely imaginary with a positive imaginary part, c.f. (2.4). Therefore, while the operator $L_{0}^{-1}$, which factors into the forward solve (see (3.5)), is exponentially smoothing, the operator $L_{0}$ amplifies Fourier coefficients of large index exponentially. 


\subsection{Expansions: Dirichlet-Neumann Operators}

Consider now the DNO $G$ which maps the surface Dirichlet data $U$ to the surface normal derivative $U^{\prime}$. We now (slightly) redefine the function

$$
u_{p}(x, y)=e^{i\left(\alpha_{p} x+\beta_{u, p} y\right)}
$$

which again satisfies Helmholtz's equation and the outgoing wave condition in the upper material. We can insert this into the definition of the operator $G$ giving

$$
G(g)\left[u_{p}(x, g(x))\right]=\left(\partial_{y} u_{p}\right)(x, g(x))-\left(\partial_{x} g\right)\left(\partial_{x} u_{p}\right)(x, g(x)),
$$

or

$$
G(g)\left[e^{i\left(\alpha_{p} x+\beta_{u, p} g(x)\right)}\right]=\left(i \beta_{u, p}-\left(\partial_{x} g\right) i \alpha_{p}\right) e^{i\left(\alpha_{p} x+\beta_{u, p} g(x)\right)} .
$$

Again setting $g(x)=\varepsilon f(x)$, and expanding $G$ and the exponentials in Taylor series gives

$$
\begin{aligned}
\left(\sum_{n=0}^{\infty} \varepsilon^{n} G_{n}(f)\right)\left[e^{i \alpha_{p} x} \sum_{m=0}^{\infty} F_{m}(x)\left(i \beta_{u, p}\right)^{m} \varepsilon^{m}\right] & =i \beta_{u, p} e^{i \alpha_{p} x} \sum_{n=0}^{\infty} F_{n}(x)\left(i \beta_{u, p}\right)^{n} \varepsilon^{n} \\
& -\varepsilon\left(\partial_{x} f\right)\left(i \alpha_{p}\right) e^{i \alpha_{p} x} \sum_{n=0}^{\infty} F_{n}(x)\left(i \beta_{u, p}\right)^{n} \varepsilon^{n} .
\end{aligned}
$$

At order $\mathcal{O}\left(\varepsilon^{0}\right)$ we find

$$
G_{0}\left[e^{i \alpha_{p} x}\right]=\left(i \beta_{u, p}\right) e^{i \alpha_{p} x}=\left(i \beta_{u, D}\right) e^{i \alpha_{p} x}
$$

or

$$
G_{0}[\xi]=\left(i \beta_{u, D}\right) \xi
$$

At order $\mathcal{O}\left(\varepsilon^{n}\right)$ we obtain

$$
\sum_{m=0}^{n} G_{m}\left[F_{n-m}\left(i \beta_{u, p}\right)^{n-m} e^{i \alpha_{p} x}\right]=F_{n}(x)\left(i \beta_{u, p}\right)^{n+1} e^{i \alpha_{p} x}-\left(\partial_{x} f\right) F_{n-1}(x)\left(i \alpha_{p}\right)\left(i \beta_{u, p}\right)^{n-1} e^{i \alpha_{p} x}
$$

or

$$
\begin{aligned}
G_{n}\left[e^{i \alpha_{p} x}\right]=\left\{F_{n}(x)\left(i \beta_{u, p}\right)^{2}-\left(\partial_{x} f\right) F_{n-1}(x)\left(i \alpha_{p}\right)\right\} & \left(i \beta_{u, p}\right)^{n-1} e^{i \alpha_{p} x} \\
& -\sum_{m=0}^{n-1} G_{m}\left[F_{n-m}\left(i \beta_{u, p}\right)^{n-m} e^{i \alpha_{p} x}\right] .
\end{aligned}
$$

Since

$$
\alpha_{p}^{2}+\beta_{u, p}^{2}=k_{u}^{2}
$$

we have

$$
\left(i \alpha_{p}\right)^{2}+\left(i \beta_{u, p}\right)^{2}=-k_{u}^{2}
$$

and

$$
\left(i \beta_{u, p}\right)^{2}=-k_{u}^{2}-\left(i \alpha_{p}\right)^{2} .
$$


Thus

$$
\begin{aligned}
G_{n}\left[e^{i \alpha_{p} x}\right]= & \left\{-k_{u}^{2} F_{n}(x)-F_{n}(x)\left(i \alpha_{p}\right)^{2}-\left(\partial_{x} f\right) F_{n-1}(x)\left(i \alpha_{p}\right)\right\}\left(i \beta_{u, p}\right)^{n-1} e^{i \alpha_{p} x} \\
& -\sum_{m=0}^{n-1} G_{m}\left[F_{n-m}\left(i \beta_{u, p}\right)^{n-m} e^{i \alpha_{p} x}\right] \\
= & -k_{u}^{2} F_{n}(x)\left(i \beta_{u, D}\right)^{n-1} e^{i \alpha_{p} x}-\partial_{x}\left[F_{n}(x) \partial_{x}\left(i \beta_{u, D}\right)^{n-1} e^{i \alpha_{p} x}\right] \\
& -\sum_{m=0}^{n-1} G_{m}\left[F_{n-m}\left(i \beta_{u, D}\right)^{n-m} e^{i \alpha_{p} x}\right],
\end{aligned}
$$

where we have used

$$
\partial_{x} e^{i \alpha_{p} x}=\left(i \alpha_{p}\right) e^{i \alpha_{p} x}
$$

Finally,

$$
G_{n}[\xi]=-k_{u}^{2} F_{n}(x)\left(i \beta_{u, D}\right)^{n-1} \xi-\partial_{x}\left[F_{n}(x) \partial_{x}\left(i \beta_{u, D}\right)^{n-1} \xi\right]-\sum_{m=0}^{n-1} G_{m}\left[F_{n-m}\left(i \beta_{u, D}\right)^{n-m} \xi\right] .
$$

In particular, for use in $\S 4.1$,

$$
\begin{aligned}
G_{1}[\xi] & =-k_{u}^{2} f \xi-\partial_{x}\left[f \partial_{x} \xi\right]-G_{0}\left[f\left(i \beta_{u, D}\right) \xi\right] \\
& =-k_{u}^{2} f \xi-\partial_{x}\left[f \partial_{x} \xi\right]-G_{0}\left[f G_{0} \xi\right] .
\end{aligned}
$$

In an exactly analogous fashion, consider the DNO $H$ which maps the surface Dirichlet data $V$ to the surface normal derivative $V^{\prime}$. Specify the function

$$
v_{p}(x, y)=e^{i\left(\alpha_{p} x-\beta_{v, p} y\right)}
$$

which satisfies Helmholtz's equation and the outgoing wave condition in the lower material. We can insert this into the definition of the operator $H$ giving

$$
H(g)\left[v_{p}(x, g(x))\right]=\left(\partial_{y} v_{p}\right)(x, g(x))-\left(\partial_{x} g\right)\left(\partial_{x} v_{p}\right)(x, g(x)),
$$

or

$$
H(g)\left[e^{i\left(\alpha_{p} x-\beta_{v, p} g(x)\right)}\right]=\left(-i \beta_{v, p}-\left(\partial_{x} g\right) i \alpha_{p}\right) e^{i\left(\alpha_{p} x-\beta_{v, p} g(x)\right)} .
$$

Once again setting $g(x)=\varepsilon f(x)$, and expanding $H$ and the exponentials in Taylor series gives

$$
\begin{aligned}
\left(\sum_{n=0}^{\infty} \varepsilon^{n} H_{n}(f)\right)\left[e^{i \alpha_{p} x} \sum_{m=0}^{\infty} F_{m}(x)\left(-i \beta_{v, p}\right)^{m} \varepsilon^{m}\right] & =-i \beta_{v, p} e^{i \alpha_{p} x} \sum_{n=0}^{\infty} F_{n}(x)\left(-i \beta_{v, p}\right)^{n} \varepsilon^{n} \\
& -\varepsilon\left(\partial_{x} f\right)\left(i \alpha_{p}\right) e^{i \alpha_{p} x} \sum_{n=0}^{\infty} F_{n}(x)\left(-i \beta_{v, p}\right)^{n} \varepsilon^{n} .
\end{aligned}
$$

At order $\mathcal{O}\left(\varepsilon^{0}\right)$ we find

$$
H_{0}\left[e^{i \alpha_{p} x}\right]=-\left(i \beta_{v, p}\right) e^{i \alpha_{p} x}=-\left(i \beta_{v, D}\right) e^{i \alpha_{p} x}
$$

or

$$
H_{0}[\xi]=-\left(i \beta_{v, D}\right) \xi
$$


At order $\mathcal{O}\left(\varepsilon^{n}\right)$ we obtain

$$
\begin{aligned}
\sum_{m=0}^{n} H_{m}\left[F_{n-m}\left(-i \beta_{v, p}\right)^{n-m} e^{i \alpha_{p} x}\right]=F_{n}(x)\left(-i \beta_{v, p}\right)^{n+1} e^{i \alpha_{p} x} & \\
& -\left(\partial_{x} f\right) F_{n-1}(x)\left(i \alpha_{p}\right)\left(-i \beta_{v, p}\right)^{n-1} e^{i \alpha_{p} x}
\end{aligned}
$$

or

$$
\begin{aligned}
H_{n}\left[e^{i \alpha_{p} x}\right]=\left\{F_{n}(x)\left(-i \beta_{v, p}\right)^{2}-\left(\partial_{x} f\right) F_{n-1}(x)\left(i \alpha_{p}\right)\right\} & \left(-i \beta_{v, p}\right)^{n-1} e^{i \alpha_{p} x} \\
& -\sum_{m=0}^{n-1} H_{m}\left[F_{n-m}\left(-i \beta_{v, p}\right)^{n-m} e^{i \alpha_{p} x}\right] .
\end{aligned}
$$

As before

$$
\left(-i \beta_{v, p}\right)^{2}=-k_{v}^{2}-\left(-i \alpha_{p}\right)^{2}=-k_{v}^{2}-\left(i \alpha_{p}\right)^{2} .
$$

Thus

$$
\begin{aligned}
H_{n}\left[e^{i \alpha_{p} x}\right]= & \left\{-k_{v}^{2} F_{n}(x)-F_{n}(x)\left(i \alpha_{p}\right)^{2}-\left(\partial_{x} f\right) F_{n-1}(x)\left(i \alpha_{p}\right)\right\}\left(-i \beta_{v, p}\right)^{n-1} e^{i \alpha_{p} x} \\
& -\sum_{m=0}^{n-1} H_{m}\left[F_{n-m}\left(-i \beta_{v, p}\right)^{n-m} e^{i \alpha_{p} x}\right] \\
= & -k_{v}^{2} F_{n}(x)\left(-i \beta_{v, D}\right)^{n-1} e^{i \alpha_{p} x}-\partial_{x}\left[F_{n}(x) \partial_{x}\left(-i \beta_{v, D}\right)^{n-1} e^{i \alpha_{p} x}\right] \\
& -\sum_{m=0}^{n-1} H_{m}\left[F_{n-m}\left(-i \beta_{v, D}\right)^{n-m} e^{i \alpha_{p} x}\right] .
\end{aligned}
$$

Finally,

$$
\begin{aligned}
H_{n}[\xi]=-k_{v}^{2} F_{n}(x)\left(-i \beta_{v, D}\right)^{n-1} \xi-\partial_{x}\left[F_{n}(x) \partial_{x}(\right. & \left.\left.-i \beta_{v, D}\right)^{n-1} \xi\right] \\
& -\sum_{m=0}^{n-1} H_{m}\left[F_{n-m}\left(-i \beta_{v, D}\right)^{n-m} \xi\right] .
\end{aligned}
$$

In particular, again for use in $\S 4.1$,

$$
\begin{aligned}
H_{1}[\xi] & =-k_{v}^{2} f \xi-\partial_{x}\left[f \partial_{x} \xi\right]-H_{0}\left[f\left(-i \beta_{v, D}\right) \xi\right] \\
& =-k_{v}^{2} f \xi-\partial_{x}\left[f \partial_{x} \xi\right]-H_{0}\left[f H_{0} \xi\right] .
\end{aligned}
$$

\subsection{Forward Solve: Flat Interface}

With formulas for the operators now in place we can utilize formulas (3.5) and (3.6) to find approximations to the $\tilde{u}_{n}$ and form

$$
\tilde{u}^{N}(x ; \varepsilon):=\sum_{n=0}^{N} \tilde{u}_{n}(x) \varepsilon^{n} .
$$

Before beginning we point out that the relevant Fourier multipliers (e.g., $i \beta_{v, D}$ ) have a particularly simple action on the single mode $e^{i \alpha x}$. For example, since

$$
e^{i \alpha x}=\sum_{p=-\infty}^{\infty} d_{p} e^{i \alpha_{p} x}, \quad d_{p}=\left\{\begin{array}{ll}
1 & p=0 \\
0 & p \neq 0
\end{array},\right.
$$


we have

$$
i \beta_{v, D}\left[e^{i \alpha x}\right]=i \beta_{v, D}\left[\sum_{p=-\infty}^{\infty} d_{p} e^{i \alpha_{p} x}\right]=\sum_{p=-\infty}^{\infty}\left(i \beta_{v, p}\right) d_{p} e^{i \alpha_{p} x}=i \beta_{v} e^{i \alpha x}
$$

Returning to our solution algorithm, (3.5) can now be written as

$$
\begin{aligned}
\tilde{u}_{0} & =e^{i \beta_{u, D} a}\left[\left(i \beta_{u, D}+i \beta_{v, D}\right)^{-1}\left[\left(i \beta_{u}\right) e^{i \alpha x}+i \beta_{v, D}\left[-e^{i \alpha x}\right]\right]\right. \\
& =e^{i \beta_{u, D} a}\left[\left(i \beta_{u, D}+i \beta_{v, D}\right)^{-1}\left[\left(i \beta_{u}-i \beta_{v}\right)\right] e^{i \alpha x}\right] \\
& =e^{i \beta_{u, D} a}\left[\frac{\left(i \beta_{u}-i \beta_{v}\right)}{\left(i \beta_{u}+i \beta_{v}\right)} e^{i \alpha x}\right] \\
& =e^{i \beta_{u} a} \frac{\left(i \beta_{u}-i \beta_{v}\right)}{\left(i \beta_{u}+i \beta_{v}\right)} e^{i \alpha x}
\end{aligned}
$$

which is, of course, the exact solution in the flat interface $(\varepsilon=0)$ case, and recovers the plane-wave reflection coefficients.

Remark. We note that in this simple flat-interface case

$$
\psi_{0}=G_{0}\left[\zeta_{0}\right]
$$

so that (3.5) simplifies to

$$
\tilde{u}_{0}=L_{0}^{-1}\left[\left(G_{0}-H_{0}\right)^{-1}\left[\left(G_{0}-H_{0}\right)\left[\zeta_{0}\right]\right]\right]=L_{0}^{-1}\left[\zeta_{0}\right]
$$

as expected.

Remark. We point out here that this formula can be used also as a very primitive inverse problem solver: If we specify the incident radiation (in particular $\beta_{u}$ ) and measure the far-field pattern $\tilde{u}_{0}$ at the known plane $y=a$, then (3.13) can be solved for $\beta_{v}$ which gives very rough material properties of the lower layer. Notice that (3.13) demands that $\tilde{u}_{0}$ have the rather trivial Fourier series

$$
\tilde{u}_{0}(x)=\tilde{u}_{0,0} e^{i \alpha},
$$

but, given this, one can use (3.13) to deduce that

$$
\beta_{v}=\beta_{u}\left(\frac{e^{i \beta_{u} a}-\tilde{u}_{0,0}}{e^{i \beta_{u} a}+\tilde{u}_{0,0}}\right)
$$

\subsection{Numerical Results for a General Interface}

To briefly test this new algorithm for the forward problem we select a configuration with physical parameters

$$
\alpha=0.1, \quad \beta_{u}=1.1, \quad \beta_{v}=5.5,
$$

c.f. $(2.2 \mathrm{f}) \&(2.2 \mathrm{~g})$, with a $d=2 \pi$-periodic layer interface shaped by

$$
g(x)=\varepsilon f(x)=\varepsilon e^{\cos (2 x)},
$$

and "far-field" $\tilde{u}$ at $a=1$. To compute an "exact solution" we utilize the Method of Field Expansions (FE) [BR93a] as implemented by the authors in the recent publication [MN10]. 


\begin{tabular}{lll}
$N$ & Absolute $L^{\infty}$ Error & Relative $L^{\infty}$ Error \\
\hline 0 & 0.000196114 & 0.000294154 \\
1 & $4.51806 \times 10^{-8}$ & $6.77671 \times 10^{-8}$ \\
2 & $3.22802 \times 10^{-10}$ & $4.84176 \times 10^{-10}$ \\
3 & $3.28269 \times 10^{-10}$ & $4.92377 \times 10^{-10}$ \\
4 & $3.2827 \times 10^{-10}$ & $4.92377 \times 10^{-10}$
\end{tabular}

Table 1: Absolute and relative $L^{\infty}$ errors in approximation of the far-field pattern $\tilde{u}$ at $a=1$. Physical parameters: $\alpha=0.1, \beta_{u}=1.1, \beta_{v}=5.5, d=2 \pi, a=1$; numerical parameter: $N_{x}=32$.

While the methods are related (both are spectral collocation Boundary Perturbation approaches), they are not identical and one provides an excellent test for the other. For the configuration mentioned above and $\varepsilon=0.0001$ we performed a numerical simulation using the FE approach with $N_{x}=128$ collocation points and $N=40$ Taylor orders (Taylor summation was used); please see [MN10] for more details regarding the algorithm and these parameters.

In Table 1 we present results of a numerical implementation of (3.5) \& (3.6) to deliver (3.12), reporting perturbation order versus absolute and relative errors. Here we notice the very stable and rapid (exponential) convergence of our numerical approximation to the "exact solution" provided by the FE method.

\section{Inverse Problem}

Our real goal in this paper is to devise a technique for recovering the layer interface, $g(x)$, from surface measurements. In this initial contribution we propose as given data the incident radiation,

$$
u^{i}(x, y)=e^{i \alpha x-i \beta_{u} y}
$$

(which includes the material properties of the upper layer through $\beta_{u}$ ), the "far field pattern," $\tilde{u}(x)$ at all values of $x$, and the most basic material properties of the lower layer: $\beta_{v}$ (which we assume can be recovered from (3.14) or some other method).

\subsection{Iteration-Free Linear Model}

With these constraints in mind, consider the forward problem (3.4), and suppose that the unknown interface can be expressed as $g(x)=\varepsilon f(x)$. In this case we have

$$
\left(G_{0}+\varepsilon G_{1}-H_{0}-\varepsilon H_{1}\right)\left[\left(L_{0}+\varepsilon L_{1}\right)[\tilde{u}]\right]-\psi_{0}-\varepsilon \psi_{1}+\left(H_{0}+\varepsilon H_{1}\right)\left[\zeta_{0}+\varepsilon \zeta_{1}\right]=\mathcal{O}\left(\varepsilon^{2}\right) .
$$

More precisely, and making the $f$ dependence explicit, we have

$$
\begin{aligned}
\left(G_{0}-H_{0}\right) L_{0}[\tilde{u}]+\varepsilon\left(G_{0}-\right. & \left.H_{0}\right) L_{1}(f)[\tilde{u}]+\varepsilon\left(G_{1}(f)-H_{1}(f)\right) L_{0}[\tilde{u}] \\
& -\psi_{0}-\varepsilon \psi_{1}(f)+H_{0}\left[\zeta_{0}\right]+\varepsilon H_{1}(f)\left[\zeta_{0}\right]+\varepsilon H_{0}\left[\zeta_{1}(f)\right]=\mathcal{O}\left(\varepsilon^{2}\right) .
\end{aligned}
$$

For a first algorithm we ignore the $\mathcal{O}\left(\varepsilon^{2}\right)$ terms and gather the $\mathcal{O}(1)$ and $\mathcal{O}(\varepsilon)$ terms separately

$$
Q_{0}(\tilde{u})+\varepsilon Q_{1}(\tilde{u})[f]=0,
$$


where

$$
\begin{aligned}
Q_{0}(\tilde{u}):= & \left(G_{0}-H_{0}\right) L_{0}[\tilde{u}]-\psi_{0}+H_{0}\left[\zeta_{0}\right] \\
Q_{1}(\tilde{u})[f]:= & \left(G_{0}-H_{0}\right) L_{1}(f)[\tilde{u}]+\left(G_{1}(f)-H_{1}(f)\right) L_{0}[\tilde{u}] \\
& -\psi_{1}(f)+H_{1}(f)\left[\zeta_{0}\right]+H_{0}\left[\zeta_{1}(f)\right] .
\end{aligned}
$$

The operator $Q_{1}(\cdot)[\tilde{u}]$ is linear in $f$, though in a rather implicit way, and we propose the following solution formula:

$$
\tilde{g}=-\left\{Q_{1}(\cdot)[\tilde{u}]\right\}^{-1} Q_{0}[\tilde{u}],
$$

where $\tilde{g} \approx g$. Notice that this approach is "linear" (i.e., terms of order two and higher were ignored) and the unique solution can be found rather directly (without iteration) by simply inverting the linear operator (represented as a matrix in a numerical simulation), $Q_{1}(\cdot)(\tilde{u})$. Remark. As we mentioned earlier (§ 3.3), the operators $L_{0}$ and $L_{1}=f\left(i \beta_{u, D}\right) L_{0},(3.9)$, are ill-conditioned resulting in potentially unstable numerics. However, such ill-conditioning is a standard feature of inverse problems [CK98] and it is to be expected in such algorithms.

\subsection{Iterative Nonlinear Model}

To devise a second, and hopefully more accurate approach, we return to the forward problem (3.4) and again suppose that the unknown interface can be expressed as $g(x)=\varepsilon f(x)$. Now,

$$
Q_{0}(\tilde{u})+\varepsilon Q_{1}(\tilde{u})[f]+\sum_{n=2}^{N} \varepsilon^{n} Q_{n}(\tilde{u}, f)=\mathcal{O}\left(\varepsilon^{N+1}\right),
$$

where

$$
Q_{n}(\tilde{u}, f)=\sum_{m=0}^{n}\left(G_{n-m}(f)-H_{n-m}(f)\right)\left[L_{m}(f)[\tilde{u}]\right]-\psi_{n}(f)+\sum_{m=0}^{n} H_{n-m}(f)\left[\zeta_{m}(f)\right] .
$$

A natural algorithm which suggests itself is to combine the higher accuracy of the expansion (4.3) for $N>1$ with the ease of inversion of (4.2); thus we drop the $\mathcal{O}\left(\varepsilon^{N+1}\right)$ term in (4.3), mark the linear (in $\varepsilon$ ) term with iteration number $k+1$, and all other terms with iteration number $k$ resulting in the Picard iteration [BF97, AH01]

$$
\tilde{g}^{k+1}=-\left\{Q_{1}(\cdot)[\tilde{u}]\right\}^{-1}\left[Q_{0}[\tilde{u}]+\sum_{n=2}^{N} Q_{n}\left(\tilde{u}, \tilde{g}^{k}\right)\right] .
$$

Note that in the case $N=1$ this becomes our linear algorithm (4.2). However, in contrast with (4.2), this new method is "nonlinear" (as we now retain quadratic and higher terms) and requires an iteration scheme for its solution. As with any iterative scheme it is of paramount importance to select a good initial guess. For this we recommend using the linear approximation, (4.2),

$$
\tilde{g}^{0}=-\left\{Q_{1}(\cdot)[\tilde{u}]\right\}^{-1} Q_{0}[\tilde{u}] .
$$

\subsection{Results}

We now demonstrate the capabilities of our new algorithms with a sequence of numerical studies. To begin, we consider the analytic and $d=2 \pi$-periodic profile

$$
g(x)=\varepsilon e^{\cos (2 x)}
$$




\begin{tabular}{lll}
$\varepsilon$ & Absolute $L^{\infty}$ Error & Relative $L^{\infty}$ Error \\
\hline 0.001 & $3.40341 \times 10^{-6}$ & 0.00125205 \\
0.002 & $1.35404 \times 10^{-5}$ & 0.00249062 \\
0.003 & $3.02975 \times 10^{-5}$ & 0.00371528 \\
0.004 & $5.35726 \times 10^{-5}$ & 0.00492706 \\
0.005 & $8.32629 \times 10^{-5}$ & 0.00612614 \\
0.006 & 0.00011928 & 0.00731342 \\
0.007 & 0.000161528 & 0.008489 \\
0.008 & 0.000209926 & 0.00965343 \\
0.009 & 0.000264389 & 0.010807 \\
0.01 & 0.000324838 & 0.0119501
\end{tabular}

Table 2: Absolute and relative $L^{\infty}$ errors in approximation of the analytic profile $y=$ $\varepsilon e^{\cos (2 x)},(4.5)$, using the exact linear model, (4.2), for reconstruction. Physical parameters: $\alpha=0, \beta_{u}=1.1, \beta_{v}=5.5, d=2 \pi, a=1$; numerical parameters: $N_{x}=32, N_{\text {forward }}=10$.

\begin{tabular}{llll}
$\varepsilon$ & Number of Iterations & Absolute $L^{\infty}$ Error & Relative $L^{\infty}$ Error \\
\hline 0.001 & 4 & $1.21923 \times 10^{-9}$ & $4.48531 \times 10^{-7}$ \\
0.002 & 5 & $1.05361 \times 10^{-9}$ & $1.938 \times 10^{-7}$ \\
0.003 & 6 & $1.50681 \times 10^{-9}$ & $1.84775 \times 10^{-7}$ \\
0.004 & 7 & $3.99985 \times 10^{-9}$ & $3.67865 \times 10^{-7}$ \\
0.005 & 8 & $7.41919 \times 10^{-9}$ & $5.45873 \times 10^{-7}$ \\
0.006 & 9 & $2.03556 \times 10^{-8}$ & $1.24807 \times 10^{-6}$ \\
0.007 & 10 & $4.26912 \times 10^{-8}$ & $2.2436 \times 10^{-6}$ \\
0.008 & 11 & $8.29894 \times 10^{-8}$ & $3.81626 \times 10^{-6}$ \\
0.009 & 12 & $1.50113 \times 10^{-7}$ & $6.13594 \times 10^{-6}$ \\
0.01 & 13 & $2.56547 \times 10^{-7}$ & $9.43782 \times 10^{-6}$
\end{tabular}

Table 3: Absolute and relative $L^{\infty}$ errors in approximation of the analytic profile $y=$ $\varepsilon e^{\cos (2 x)},(4.5)$, using the nonlinear model, (4.4), for reconstruction. Physical parameters: $\alpha=0, \beta_{u}=1.1, \beta_{v}=5.5, d=2 \pi, a=1$; numerical parameters: $N_{x}=32, N_{\text {forward }}=10$, $\tau=10^{-8}, N_{\text {inverse }}=4$.

(see Figure 1) as the shape of the interface between two materials with $k_{u}=1.1$ and $k_{v}=5.5$. Utilizing our algorithm for the forward problem, (3.6), we generate a far-field pattern $\tilde{u}$ (with $N_{x}=32$ equally spaced gridpoints and $N=N_{\text {forward }}=10$ Taylor orders). Using the "linear model" (4.2) we produce the approximation $\tilde{g}_{0}$ and in Table 2 report on the absolute and relative supremum norm errors in the recovery of $g$ for various values of $\varepsilon$. We note the rapid rate of convergence as $\varepsilon \rightarrow 0$ which is repeated for all of the profiles considered here. Additionally, we use the nonlinear iterative approach (4.4) to approximate $g$ (with initial guess $\tilde{g}_{0}, N=N_{\text {inverse }}=4$, and tolerance $\tau=10^{-8}$ for the iteration) and display these absolute and relative errors in Table 3 . In these we see not only the rapid and stable convergence of both of our new approaches to the specified boundary shape $g(x)$, but also the highly advantageous nature of the nonlinear iteration scheme which can generate three to four more digits of accuracy with only a modest (4 to 13) number of iterations. 


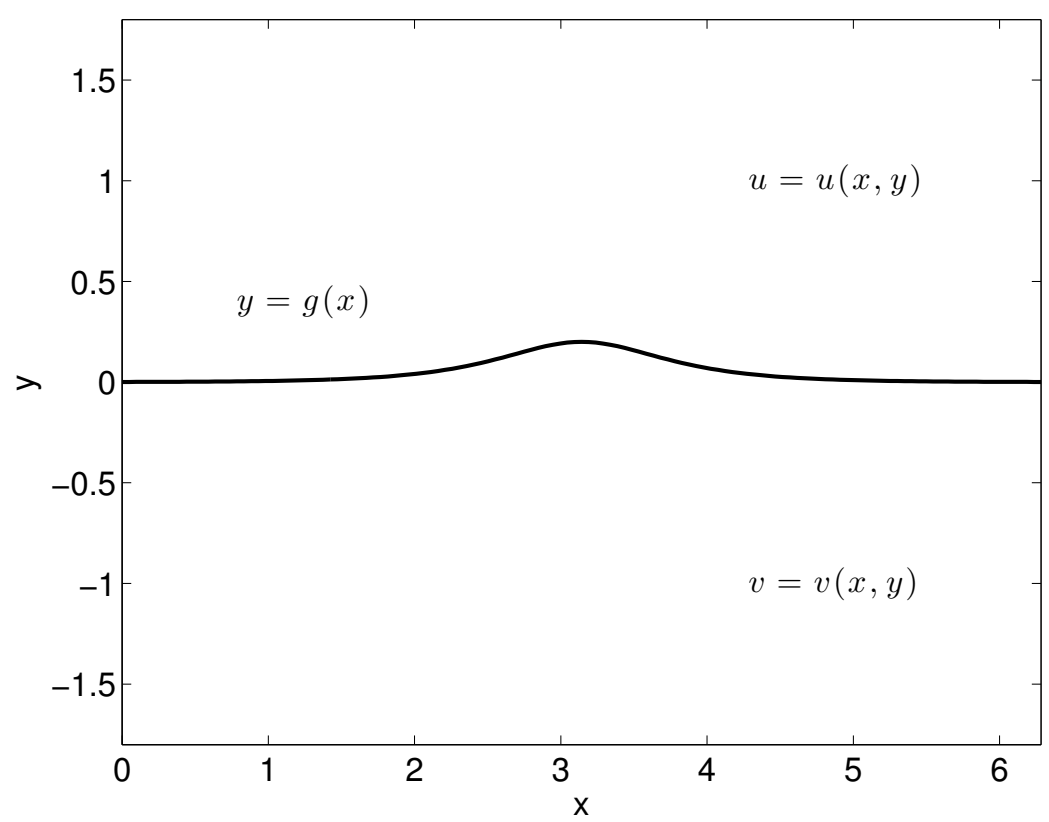

Figure 2: Problem configuration with layer boundary (solid line); here $g(x)=0.2 \operatorname{sech}(2(x-$ $\pi))$.

We now move on to two other profiles, one meant to resemble a Gaussian pulse

$$
g_{G}(x)=\varepsilon \operatorname{sech}(b(x-d / 2))
$$

and another meant to model a smoothed bar

$$
g_{B}(x)=\varepsilon[\tanh (b((x-d / 2)+c))-\tanh (b(x-(d / 2)-c))] ;
$$

see Figures $2 \& 3$. For these interfaces we select $\alpha=0.2$, and materials such that $\beta_{u}=1.3$ and $\beta_{v}=6.8$ (so that $k_{u} \approx 1.3153$ and $k_{v} \approx 6.8029$ ). Once again we produce a far-field pattern using our forward algorithm, (3.6), with $N_{x}=32$ equally spaced gridpoints and $N_{\text {forward }}=10$ Taylor orders. With the "linear model" (4.2) we produce the approximation $\tilde{g}_{0}$ and in Tables $4 \& 6$ report on the absolute and relative supremum norm errors in the recovery of $g_{G}$ and $g_{B}$, respectively. Additionally, we use the nonlinear iterative approach (4.4) to approximate $g_{G}$ and $g_{B}$ (with initial guess $\tilde{g}_{0}$, degree of nonlinearity $N_{\text {inverse }}=4$, and tolerance $\tau=10^{-8}$ ) and display these absolute and relative errors in Tables $5 \& 7$. As with the analytic profile above we note both the rapid convergence of our approach, and the truly superior accuracy one can achieve with the nonlinear iterative methodology.

\section{References}

[AH01] Kendall Atkinson and Weimin Han. Theoretical numerical analysis, volume 39 of Texts in Applied Mathematics. Springer-Verlag, New York, 2001. A functional analysis framework.

[AKY06] Ibrahim Akduman, Rainer Kress, and Ali Yapar. Iterative reconstruction of dielectric rough surface profiles at fixed frequency. Inverse Problems, 22(3):939$954,2006$. 


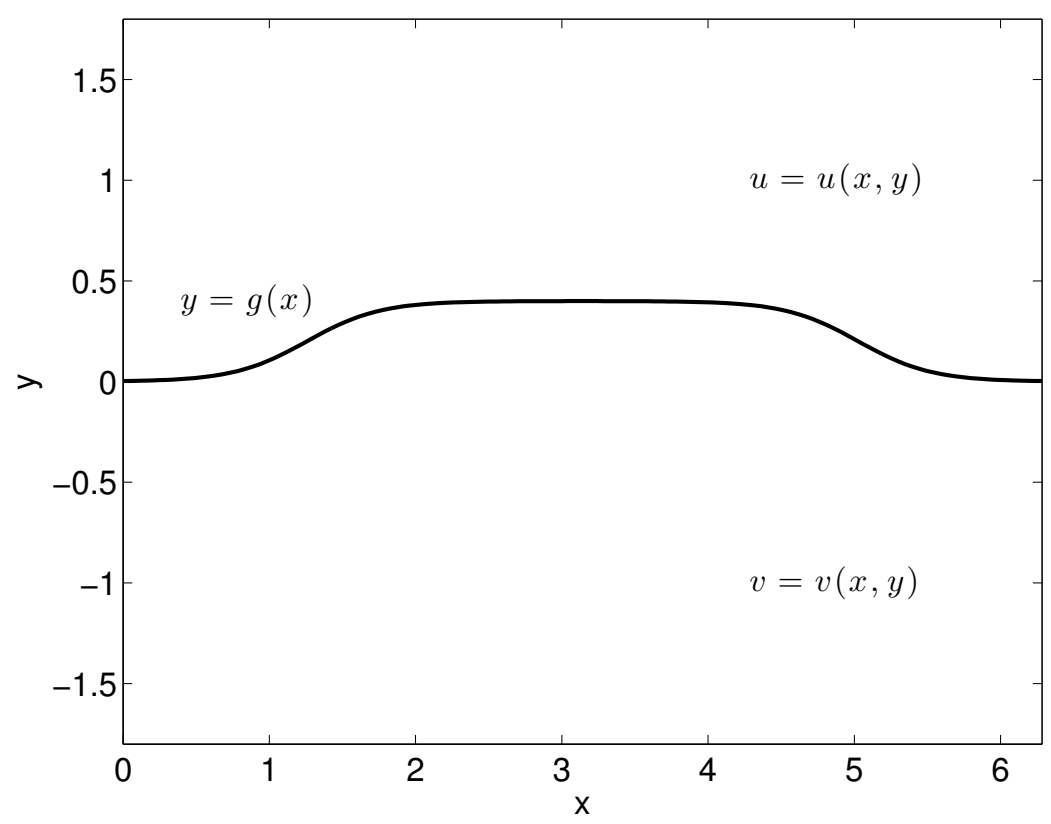

Figure 3: Problem configuration with layer boundary (solid line); here $g(x)=$ $0.2[\tanh (2((x-\pi)+3 \pi / 5))-\tanh (2(x-\pi)-3 \pi / 5))]$.

\begin{tabular}{lll}
$\varepsilon$ & Absolute $L^{\infty}$ Error & Relative $L^{\infty}$ Error \\
\hline 0.001 & $3.59224 \times 10^{-7}$ & 0.000359224 \\
0.002 & $1.43428 \times 10^{-6}$ & 0.00071714 \\
0.003 & $3.22159 \times 10^{-6}$ & 0.00107386 \\
0.004 & $5.71794 \times 10^{-6}$ & 0.00142949 \\
0.005 & $8.92124 \times 10^{-6}$ & 0.00178425 \\
0.006 & $1.28265 \times 10^{-5}$ & 0.00213776 \\
0.007 & $1.74317 \times 10^{-5}$ & 0.00249024 \\
0.008 & $2.2733 \times 10^{-5}$ & 0.00284163 \\
0.009 & $2.87292 \times 10^{-5}$ & 0.00319213 \\
0.01 & $3.54161 \times 10^{-5}$ & 0.00354161
\end{tabular}

Table 4: Absolute and relative $L^{\infty}$ errors in approximation of the Gaussian profile $y=$ $\varepsilon \operatorname{sech}(b x)(b=2)$, (4.6), using the exact linear model, (4.2), for reconstruction. Physical parameters: $\alpha=0.2, \beta_{u}=1.3, \beta_{v}=6.8, d=2 \pi, a=1$; numerical parameters: $N_{x}=32$, $N_{\text {forward }}=10$. 


\begin{tabular}{llll}
$\varepsilon$ & Number of Iterations & Absolute $L^{\infty}$ Error & Relative $L^{\infty}$ Error \\
\hline 0.001 & 3 & $5.69307 \times 10^{-10}$ & $5.69307 \times 10^{-7}$ \\
0.002 & 4 & $5.20979 \times 10^{-10}$ & $2.6049 \times 10^{-7}$ \\
0.003 & 4 & $5.9729 \times 10^{-10}$ & $1.99097 \times 10^{-7}$ \\
0.004 & 5 & $4.01966 \times 10^{-10}$ & $1.00492 \times 10^{-7}$ \\
0.005 & 5 & $8.17942 \times 10^{-10}$ & $1.63588 \times 10^{-7}$ \\
0.006 & 5 & $6.83363 \times 10^{-10}$ & $1.13894 \times 10^{-7}$ \\
0.007 & 6 & $6.81667 \times 10^{-10}$ & $9.73811 \times 10^{-8}$ \\
0.008 & 6 & $5.42917 \times 10^{-10}$ & $6.78646 \times 10^{-8}$ \\
0.009 & 6 & $1.05607 \times 10^{-9}$ & $1.17341 \times 10^{-7}$ \\
0.01 & 7 & $2.16823 \times 10^{-9}$ & $2.16823 \times 10^{-7}$
\end{tabular}

Table 5: Absolute and relative $L^{\infty}$ errors in approximation of the Gaussian profile $y=$ $\varepsilon \operatorname{sech}(b x)(b=2),(4.6)$, using the nonlinear model, (4.4), for reconstruction. Physical parameters: $\alpha=0.2, \beta_{u}=1.3, \beta_{v}=6.8, d=2 \pi, a=1$; numerical parameters: $N_{x}=32$, $N_{\text {forward }}=10, \tau=10^{-8}, N_{\text {inverse }}=4$.

\begin{tabular}{lll}
$\varepsilon$ & Absolute $L^{\infty}$ Error & Relative $L^{\infty}$ Error \\
\hline 0.001 & $4.44073 \times 10^{-7}$ & 0.000222273 \\
0.002 & $1.77819 \times 10^{-6}$ & 0.000445019 \\
0.003 & $4.00301 \times 10^{-6}$ & 0.000667879 \\
0.004 & $7.12105 \times 10^{-6}$ & 0.000891079 \\
0.005 & $1.11326 \times 10^{-5}$ & 0.00111445 \\
0.006 & $1.60395 \times 10^{-5}$ & 0.00133805 \\
0.007 & $2.18409 \times 10^{-5}$ & 0.00156172 \\
0.008 & $2.85384 \times 10^{-5}$ & 0.00178555 \\
0.009 & $3.61323 \times 10^{-5}$ & 0.00200949 \\
0.01 & $4.46244 \times 10^{-5}$ & 0.00223359
\end{tabular}

Table 6: Absolute and relative $L^{\infty}$ errors in approximation of the bar profile $y=$ $\varepsilon[\tanh (b(x+c))-\tanh (b(x-c))](b=2, c=3 \pi / 5),(4.7)$, using the exact linear model, (4.2), for reconstruction. Physical parameters: $\alpha=0.2, \beta_{u}=1.3, \beta_{v}=6.8, d=2 \pi, a=1$; numerical parameters: $N_{x}=32, N_{\text {forward }}=10$. 


\begin{tabular}{llll}
$\varepsilon$ & Number of Iterations & Absolute $L^{\infty}$ Error & Relative $L^{\infty}$ Error \\
\hline 0.001 & 3 & $4.14524 \times 10^{-10}$ & $2.07482 \times 10^{-7}$ \\
0.002 & 4 & $2.98051 \times 10^{-10}$ & $7.4592 \times 10^{-8}$ \\
0.003 & 5 & $7.76437 \times 10^{-10}$ & $1.29544 \times 10^{-7}$ \\
0.004 & 5 & $8.70447 \times 10^{-10}$ & $1.08922 \times 10^{-7}$ \\
0.005 & 5 & $8.92073 \times 10^{-10}$ & $8.93022 \times 10^{-8}$ \\
0.006 & 6 & $9.98351 \times 10^{-10}$ & $8.32844 \times 10^{-8}$ \\
0.007 & 6 & $1.93522 \times 10^{-9}$ & $1.38377 \times 10^{-7}$ \\
0.008 & 6 & $3.51224 \times 10^{-9}$ & $2.19749 \times 10^{-7}$ \\
0.009 & 7 & $6.06607 \times 10^{-9}$ & $3.37362 \times 10^{-7}$ \\
0.01 & 7 & $1.01294 \times 10^{-8}$ & $5.07009 \times 10^{-7}$
\end{tabular}

Table 7: Absolute and relative $L^{\infty}$ errors in approximation of the bar profile $y=$ $\varepsilon[\tanh (b(x+c))-\tanh (b(x-c))](b=2, c=3 \pi / 5),(4.7)$, using the nonlinear model, (4.4), for reconstruction. Physical parameters: $\alpha=0.2, \beta_{u}=1.3, \beta_{v}=6.8, d=2 \pi, a=1$; numerical parameters: $N_{x}=32, N_{\text {forward }}=10, \tau=10^{-8}, N_{\text {inverse }}=4$.

[BF97] Richard Burden and J. Douglas Faires. Numerical analysis. Brooks/Cole Publishing Co., Pacific Grove, CA, sixth edition, 1997.

[Bou03] M Bouchon. A review of the discrete wavenumber method. Pure appl. geophys., 160(3):445-465, 2003.

[BR92] Oscar P. Bruno and Fernando Reitich. Solution of a boundary value problem for the Helmholtz equation via variation of the boundary into the complex domain. Proc. Roy. Soc. Edinburgh Sect. A, 122(3-4):317-340, 1992.

[BR93a] Oscar P. Bruno and Fernando Reitich. Numerical solution of diffraction problems: A method of variation of boundaries. J. Opt. Soc. Am. A, 10(6):1168$1175,1993$.

[BR93b] Oscar P. Bruno and Fernando Reitich. Numerical solution of diffraction problems: A method of variation of boundaries. II. Finitely conducting gratings, Padé approximants, and singularities. J. Opt. Soc. Am. A, 10(11):2307-2316, 1993.

[BR93c] Oscar P. Bruno and Fernando Reitich. Numerical solution of diffraction problems: A method of variation of boundaries. III. Doubly periodic gratings. $J$. Opt. Soc. Am. A, 10(12):2551-2562, 1993.

[CK98] David Colton and Rainer Kress. Inverse acoustic and electromagnetic scattering theory. Springer-Verlag, Berlin, second edition, 1998.

[CM85] R. Coifman and Y. Meyer. Nonlinear harmonic analysis and analytic dependence. In Pseudodifferential operators and applications (Notre Dame, Ind., 1984), pages 71-78. Amer. Math. Soc., 1985.

[CS93] Walter Craig and Catherine Sulem. Numerical simulation of gravity waves. Journal of Computational Physics, 108:73-83, 1993. 
[CSS97] Walter Craig, Ulrich Schanz, and Catherine Sulem. The modulation regime of three-dimensional water waves and the Davey-Stewartson system. Ann. Inst. Henri Poincaré, 14:615-667, 1997.

[GR87] L. Greengard and V. Rokhlin. A fast algorithm for particle simulations. J. Comput. Phys., 73(2):325-348, 1987.

[HN05] Bei Hu and David P. Nicholls. Analyticity of Dirichlet-Neumann operators on Hölder and Lipschitz domains. SIAM J. Math. Anal., 37(1):302-320, 2005.

[HN10] Bei Hu and David P. Nicholls. The domain of analyticity of DirichletNeumann operators. Proceedings of the Royal Society of Edinburgh A, 140(2):367-389, 2010.

[KFI04] K. Koketsu, H. Fujiwara, and Y. Ikegami. Finite-element simulation of seismic ground motion with a voxel mesh. Journal Pure and Applied Geophysics, 161(11-12):2183-2198, 2004.

[KT00] R. Kress and T. Tran. Inverse scattering for a locally perturbed half-plane. Inverse Problems, 16(5):1541-1559, 2000.

[KT02a] D. Komatitsch and J. Tromp. Spectral-element simulations of global seismic wave propagation-I. Validation. Geophysical Journal International, 149(2):390-412, 2002.

[KT02b] D. Komatitsch and J. Tromp. Spectral-element simulations of global seismic wave propagation-II. 3-D models, oceans, rotation, and self-gravitation. Geophysical Journal International, 150(1):303-318, 2002.

[LG11] Chorfi Lahcene and Patricia Gaitan. Reconstruction of the interface between two-layered media using far field measurements. Inverse Problems, 27(7), 2011.

[Mil91a] D. Michael Milder. An improved formalism for rough-surface scattering of acoustic and electromagnetic waves. In Proceedings of SPIE - The International Society for Optical Engineering (San Diego, 1991), volume 1558, pages 213-221. Int. Soc. for Optical Engineering, Bellingham, WA, 1991.

[Mil91b] D. Michael Milder. An improved formalism for wave scattering from rough surfaces. J. Acoust. Soc. Am., 89(2):529-541, 1991.

[Mil96a] D. Michael Milder. An improved formalism for electromagnetic scattering from a perfectly conducting rough surface. Radio Science, 31(6):1369-1376, 1996.

[Mil96b] D. Michael Milder. Role of the admittance operator in rough-surface scattering. J. Acoust. Soc. Am., 100(2):759-768, 1996.

[MN10] Alison Malcolm and David P. Nicholls. A field expansions method for scattering by periodic multilayered media. Journal of the Acoustical Society of America (to appear), 2010. 
[MRE07] P Moczo, JOA Robertsson, and L Eisner. The finite-difference time-domain method for modeling of seismic wave propagation. Advances in Geophysics, 48:421-516, 2007.

[MS91] D. Michael Milder and H. Thomas Sharp. Efficient computation of rough surface scattering. In Mathematical and numerical aspects of wave propagation phenomena (Strasbourg, 1991), pages 314-322. SIAM, Philadelphia, PA, 1991.

[MS92] D. Michael Milder and H. Thomas Sharp. An improved formalism for rough surface scattering. ii: Numerical trials in three dimensions. J. Acoust. Soc. Am., 91(5):2620-2626, 1992.

[NR01] David P. Nicholls and Fernando Reitich. A new approach to analyticity of Dirichlet-Neumann operators. Proc. Roy. Soc. Edinburgh Sect. A, 131(6):1411-1433, 2001.

[NR03] David P. Nicholls and Fernando Reitich. Analytic continuation of DirichletNeumann operators. Numer. Math., 94(1):107-146, 2003.

[NR04a] David P. Nicholls and Fernando Reitich. Shape deformations in rough surface scattering: Cancellations, conditioning, and convergence. J. Opt. Soc. Am. A, 21(4):590-605, 2004.

[NR04b] David P. Nicholls and Fernando Reitich. Shape deformations in rough surface scattering: Improved algorithms. J. Opt. Soc. Am. A, 21(4):606-621, 2004.

[NT08] David P. Nicholls and Mark Taber. Joint analyticity and analytic continuation for Dirichlet-Neumann operators on doubly perturbed domains. J. Math. Fluid Mech., 10(2):238-271, 2008.

[NT09] David P. Nicholls and Mark Taber. Detection of ocean bathymetry from surface wave measurements. Euro. J. Mech. B/Fluids, 28(2):224-233, 2009.

[Pet80] Roger Petit, editor. Electromagnetic theory of gratings. Springer-Verlag, Berlin, 1980.

[Pra90] R. Gerhard Pratt. Frequency-domain elastic wave modeling by finite differences: A tool for crosshole seismic imaging. Geophysics, 55(5):626-632, 1990.

[SSPRCP89] FJ Sanchez-Sesma, E Perez-Rocha, and S Chavez-Perez. Diffraction of elastic waves by three-dimensional surface irregularities. part II. Bulletin of the Seismological Society of America, 79(1):101-112, 1989.

[Zie77] O. C. Zienkiewicz. The Finite Element Method in Engineering Science, 3rd ed. McGraw-Hill, New York, 1977. 